OPEN ACCESS

Edited by:

Fatah Kashanchi,

George Mason University, USA

Reviewed by:

Juan M. Tomas,

University of Barcelona, Spain

Wai-Leng Lee,

Monash University Malaysia, Malaysia

*Correspondence:

Adong Shen

shenad16@hotmail.com

${ }^{\dagger}$ Co-first authors.

Specialty section:

This article was submitted to

Infectious Diseases,

a section of the journal

Frontiers in Microbiology

Received: 05 September 2016

Accepted: 23 November 2016

Published: 15 December 2016

Citation:

Li J, Sun L, Xu F, Qi H, Shen C, Jiao

W, Xiao J, Li Q, XU B and Shen A

(2016) Screening and Identification of

$A P O C 1$ as a Novel Potential

Biomarker for Differentiate of

Mycoplasma pneumoniae in Children

Front. Microbiol. 7:1961.

doi: 10.3389/fmicb.2016.01961

\section{Screening and Identification of APOC1 as a Novel Potential Biomarker for Differentiate of Mycoplasma pneumoniae in Children}

\author{
Jieqiong Li ${ }^{\dagger}$, Lin Sun ${ }^{\dagger}$, Fang Xu, Hui Qi, Chen Shen, Weiwei Jiao, Jing Xiao, Qinjing Li, \\ Baoping $\mathrm{Xu}$ and Adong Shen *
}

MOE Key Laboratory of Major Diseases in Children, National Key Discipline of Pediatrics (Capital Medical University), National Clinical Research Center for Respiratory Diseases, Beijing Key Laboratory of Pediatric Respiratory Infection Diseases, Beijing

Pediatric Research Institute, Beijing Children's Hospital, Capital Medical University, Beijing, China

Background: Although Mycoplasma pneumoniae (MP) is a common cause of community-acquired pneumonia (CAP) in children, the currently used diagnostic methods are not optimal. Proteomics is increasingly being used to study the biomarkers of infectious diseases.

Methods: Label-free quantitative proteomics and liquid chromatography-mass/mass spectrometry were used to analyze the fold change of protein expression in plasma of children with MP pneumonia (MPP), infectious disease control (IDC), and healthy control $(\mathrm{HC})$ groups. Selected proteins that can distinguish MPP from HC and IDC were further validated by enzyme-linked immunosorbent assay (ELISA).

Results: After multivariate analyses, 27 potential plasma biomarkers were identified to be expressed differently among child MPP, HC, and IDC groups. Among these proteins, SERPINA3, APOC1, ANXA6, KNTC1, and CFLAR were selected for ELISA verification. SERPINA3, APOC1, and CFLAR levels were significantly different among the three groups and the ratios were consistent with the trends of proteomics results. A comparison of MPP patients and HC showed APOC1 had the largest area under the curve (AUC) of 0.853 , with $77.6 \%$ sensitivity and $81.1 \%$ specificity. When APOC1 levels were compared between MPP and IDC patients, it also showed a relatively high AUC of 0.882 , with $77.6 \%$ sensitivity and $85.3 \%$ specificity.

Conclusion: APOC1 is a potential biomarker for the rapid and noninvasive diagnosis of MPP in children. The present finding may offer new insights into the pathogenesis and biomarker selection of MPP in children.

Keywords: plasma proteins, Mycoplasma pneumoniae, children, label-free quantitative proteomics, APOC1

\section{INTRODUCTION}

Mycoplasma pneumoniae (MP), the smallest free-living organism, is a common cause of upper and lower respiratory tract infections (Sanchez-Vargas and Gomez-Duarte, 2008). MP pneumonia (MPP) causes up to $40 \%$ of community-acquired pneumonia (CAP) in children and this is even higher ratio during epidemics. Although it is a self-limiting disease, some cases develop into 
refractory or fulminant pneumonia that can threaten the lives of children (Waites and Talkington, 2004).

The pathophysiology of MP infection is complex and the underlying molecular mechanisms are reported to be associated with many proteins. MP infection is thought to influence the expression of associated proteins, which are released into the bloodstream through different pathways (Covani et al., 2008; Sun et al., 2008; Li et al., 2014). Plasma proteins including cytokines, growth factors, extracellular matrix proteins, and other soluble mediators are essential for MP infection. In terms of pediatric MPP diagnosis, culture and serological tests are insensitive, timeconsuming, and cross-reactive in children (Daxboeck et al., 2003; Long et al., 2012); therefore, they are not appropriate for the rapid and accurate detection of MP infection in clinical practice.

In general, detecting biomarkers in the plasma is a useful auxiliary method to diagnosis disease (Chen et al., 2013; Meyer Sauteur et al., 2014; Shu et al., 2015). Recently, advances in high-throughput technologies, such as proteomics, have made the analysis of plasma proteins possible (Li et al., 2014). Proteomic analysis using a label-free protocol is increasingly being performed for biomarker selection. Based on the principle that a special mixture of plasma proteins present different characterizations, this technique has been widely used in many diseases including infectious diseases (Papadopoulos et al., 2004; Ren et al., 2004; Agranoff et al., 2006; Hodgetts et al., 2007), cancer (Engwegen et al., 2006), and vascular disease (Pinet et al., 2008; Zhang et al., 2008; Hong et al., 2009). Although many protein biomarkers of MPP have been indicated by proteomics, specific proteins that can be used to discriminate MPP from other infection diseases, especially in children, have not been fully elucidated.

In this study, we analyzed the fold change of protein expression of children with MPP, infectious disease controls (IDC), and healthy controls (HC) using label-free quantitative proteomics and liquid chromatography-mass/mass spectrometry (LC-MS/MS). Proteins identified that could distinguish MPP from $\mathrm{HC}$ and IDC were further validated by enzyme-linked immunosorbent assay (ELISA). The aim of this study was to screen potential protein biomarkers in plasma from children that could be used to distinguish MPP from HC and IDC.

\section{MATERIALS AND METHODS}

\section{Patients and Controls}

This study was performed in the Beijing Children's Hospital from November 2014 to September 2015. During the first period, a total of 20 children hospitalized with a final diagnosis of MPP confirmed in serum samples using PCR and ELISA were enrolled. Symptoms of children included fever, acute respiratory symptoms (cough, tachypnoea, difficult breathing) or both (Tamura et al., 2008; Wang et al., 2014). Seventeen other children defined as IDC were collected accordingly and had symptoms including respiratory symptoms and negative MPP immunoglobulin $(\mathrm{Ig}) \mathrm{M}(<1: 80)$ to exclude MPP. HC group subjects $(n=20)$ were recruited from children undergoing physical examination in Beijing Children's Hospital from November 2014 to May 2015. Patients with immunosuppression and those who received immunosuppressive therapy were excluded. All the groups were matched by age, gender, and ethnicity.

\section{Protein Extraction}

Human plasma with the removal of IgG, IgA, albumin, antitrypsin, haptoglobin, and transferrin, were mixed together for each group and divided into three tubes which were tested respectively. Each mixed sample was suspended with phosphate buffered saline (PBS, $50 \mu \mathrm{L}$ ), centrifuged at $10,000 \times \mathrm{g}$ for 30 min in $4^{\circ} \mathrm{C}$, and suspended in $100 \mu \mathrm{L}$ lysis buffer $(7 \mathrm{M}$ urea, 2 $M$ thiourea). After being centrifuged at 40,000 $\times$ g for $30 \mathrm{~min}$, proteins were extracted by ultrasonic sonication and precipitated with trichloroacetic acid for $30 \mathrm{~min}$ on ice. Then, samples were diluted with $50 \mathrm{mM} \mathrm{NH}_{4} \mathrm{HCO}_{3}$ to a final concentration of 0.5 $\mathrm{mg} / \mathrm{mL}$, and each sample was mixed with DTT $(5 \mu \mathrm{L}, 1 \mathrm{~mol} / \mathrm{L})$ at $37^{\circ} \mathrm{C}$ for $60 \mathrm{~min}$, diluted with IAA $(20 \mu \mathrm{L}, 1 \mathrm{~mol} / \mathrm{L})$ for 60 min in the dark, and digested with trypsin at $37^{\circ} \mathrm{C}$ for $12 \mathrm{~h}$. The digested supernatant fractions were used for LC-MS/MS analysis (Lee et al., 2015).

\section{LC-MS/MS Analysis}

Peptide mixtures were subjected to nano-liquid chromatography associated with MS for protein identification. All of the mass analyses were performed using a LC-MS/MS system, which consisted of an Agilent 1100 quaternary HPLC (Agilent, EASYnLC1000, USA) and a Q-Exactive mass spectrometer (Thermo Finnigan, Germany) with the application of a distal $180^{\circ} \mathrm{C}$ source temperature. An RP trap column (Thermo EASY column SC200, $150 \mu \mathrm{m} \times 100 \mathrm{~mm}$ ) and a C18 reverse-phase column (Thermo EASY column SC001 traps, $150 \mu \mathrm{m} \times 20 \mathrm{~mm}$ ) was used for desalting and separating samples, respectively. Mobile phase A consisted of HPLC-grade water containing $0.1 \%$ formic acid (FA), and phase B consisted of $84 \%$ HPLC-grade acetonitrile (ACN) containing $0.1 \%$ FA. The analytical separation was run at a flow rate of $400 \mathrm{nl} / \mathrm{min}$ by using a linear gradient of phase $\mathrm{B}$ as follows: $0-45 \%$ for $100 \mathrm{~min}, 45-100 \%$ for $8 \mathrm{~min}$, and $100 \%$ for 12 $\mathrm{min}$. Each LC-MS/MS analysis was repeated three times to reduce technical variation (Lee et al., 2015).

\section{Bioinformatics Analysis}

For protein identification, we used the Mascot 2.1 program (Matrix science) to search fragmentation spectra against a human database. For full MS or MS/MS spectra searches, an error of six parts per million (ppm) or $20 \mathrm{ppm}$ was set, respectively, and two missed cleavages were allowed. Peptide mass tolerance was set to $0.5 \mathrm{Da}$, fragment mass tolerance was set to $10 \mathrm{ppm}$. Variable modification was oxidation of methionine, static modification was carbamidomethylation of cysteine. Protein identification was considered valid if at least one peptide and the $p<0.05$, the proteins not satisfying these defined criteria were rejected, the threshold for accepting MS/MS spectra was false discovery rate (FDR) 0.05. The Maxquant MS Analysis Software was used to estimate the fold-changes in the level of identified proteins between the three groups.

A Web-accessible resource (Version 2.0) was used to determine the over-representation of Gene Ontology (GO) 
categories, which was classified on the basis of cellular component (CC), molecular function (MF), and biological process (BP) (Zhang et al., 2014; Wu et al., 2016). Signaling pathway analysis was performed using tools from the Kyoto Encyclopedia of Genes and Genome (KEGG) database (http:// www.genome.jp/kegg/pathway.html) (Kanehisa et al., 2004). A network model of protein interactions according to known protein-protein interactions was made using Retrieval of Interacting Genes (STRING) 9.05.

\section{ELISA}

ELISA was used to quantify the concentrations of the selected plasma proteins. Human alpha-1-antichymotrypsin precursor (SERPINA3) (RayBiotech), human kinetochore-associated protein 1 (KNTC1) (Dldevelop), human apolipoprotein C-I precursor (APOC1) (RayBiotech), human annexin A6 isoform 2 (ANXA6) (Abnova), and CASP8 and FADD-like apoptosis regulator isoform 6 (CFLAR) (Dldevelop) ELISA kits were used to measure the changes in plasma protein levels in the MPP $(n=85), \mathrm{HC}(n=95)$, and IDC $(n=75)$ groups. ELISAs were performed according to each kit's instructions.

\section{Statistical Analysis}

Each experiment was independently repeated at least three times. Data were expressed as the mean $\pm S D$ and evaluated by Student's $t$-test. $P<0.05$ was considered statistically significant.

Statistical analysis was performed using MetaboAnalyst 3.0 (Biomarker Analysis).

Classical univariate receiver operating characteristic (ROC) curve analyses to generate ROC curves, calculate the area under the curve (AUC) and 95\% confidence intervals, compute optimal cutoffs for any given feature, and to generate performance tables for sensitivity, specificity, and confidence intervals at different cutoffs were performed. ROC curve analyses were performed based on three multivariate algorithms-support vector machines, which were used to assess the diagnostic value of candidate proteins.

\section{Ethics Statement}

This research was approved by the Ethics Committee of Beijing Children's Hospital (Supplementary Table 1). Written informed consent was collected from the children or the guardians of children. In accordance with the Declaration of Helsinki and the local ethics committee requirements, the plasma collection protocol in this research was based on the following standards: (1) Potential risk of the minor discomfort was minimized by utilizing only well trained personnel for the procedures; (2) No charge; (3) Written informed consent was provided to the children or the guardians; (4) The participants had the right to decide whether or not to participate; (5) The participants in this study will not receive direct benefits from the proposed research. However, the benefits to society could be a potentially less expensive and faster way to diagnose a serious disease and begin treatment earlier than current diagnostic methods.

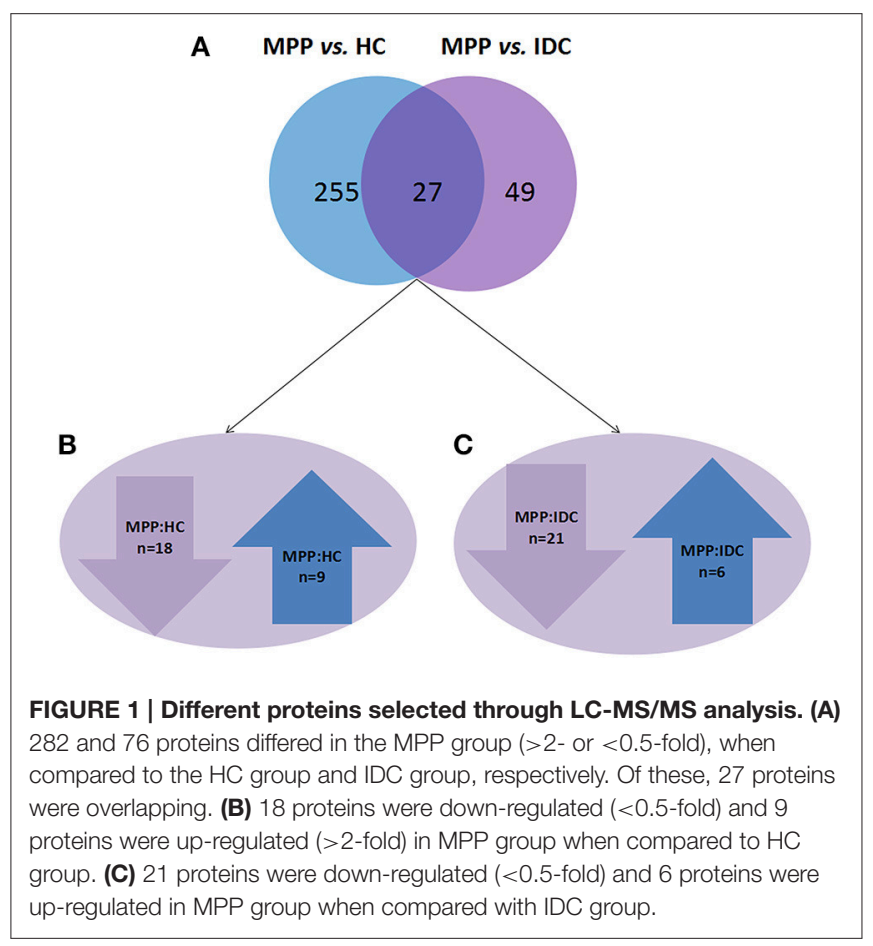

TABLE 1 | Demographic characteristic of the participants.

\begin{tabular}{|c|c|c|c|c|c|c|c|c|}
\hline & \multicolumn{4}{|c|}{ Selected period } & \multicolumn{4}{|c|}{ Verification period } \\
\hline & MPP & $\mathrm{HC}$ & IDC & $P$-value ${ }^{a}$ & MPP & $\mathrm{HC}$ & IDC & $P$-value ${ }^{a}$ \\
\hline Sample size & 20 & 20 & 17 & - & 85 & 95 & 75 & - \\
\hline \multicolumn{9}{|l|}{ Sex (\%) } \\
\hline Boy & 11 & 11 & 9 & 0.302 & 42 & 48 & 39 & 0.315 \\
\hline Girl & 9 & 9 & 8 & & 43 & 47 & 36 & \\
\hline Age (years) $^{\mathrm{b}}$ & $6.9 \pm 3.0$ & $6.3 \pm 3.5$ & $5.6 \pm 3.9$ & 0.323 & $7.0 \pm 3.0$ & $7.0 \pm 3.0$ & $6.2 \pm 5.6$ & 0.082 \\
\hline Age range (years) & $1.9-12.4$ & $3-12$ & $1-12$ & - & $1.5-15.1$ & $2.1-17.1$ & $1.9-17.2$ & - \\
\hline
\end{tabular}

a $P$-value among MPP, HC, and IDC.

${ }^{b}$ Data are presented as mean $\pm S D$. 


\section{RESULTS}

\section{Characteristics of the Study Population}

During the selected period, of 20 children with MPP enrolled in our study, 11 (55\%) were boys. The mean age of the patients in the MPP group was 6.9 years, ranging from 1.9 to 12.4 years. Among 17 children in the IDC group, the causative agents included Streptococcus pneumoniae $(n=2)$, Influenza B virus $(n=3)$, Escherichia coli $(n=3)$, Mycobacterium tuberculosis $(n=2)$, Haemophilus $(n=3)$, and Epstein-Barr virus $(n=4)$. Regarding patient demographics, no significant differences in age or gender were identified among the MPP, HC, and IDC groups.

During the verification period, $85 \mathrm{MPP}$ children, $95 \mathrm{HC}$ and 75 IDC were recruited for ELISA verification. No significant differences in age or gender were identified among these three groups (Table $\mathbf{1}$ ).

\section{Label-Free Quantitative Proteomic Analysis}

Label-free quantitative proteomics coupled with LCMS/MS analysis were used to compare samples from the three groups (MPP, HC, and IDC). Based on the LC-MS/MS data, a total of 2777 proteins were identified.

The analysis results showed that 282 and 76 proteins differed between the MPP group ( $>2$ - or $<0.5$-fold) and the HC group and IDC group, respectively (Supplementary Tables 2 and 3). Of these, 27 overlapping proteins were further analyzed: 18 proteins were down-regulated $(<0.5$-fold) and 9 proteins were up-regulated ( $>2$-fold) in the MPP group compared with the HC group. Twenty-one proteins were down-regulated $(<0.5-$ fold) and six proteins were up-regulated ( $>2$-fold) in the MPP group compared with the IDC group (Figure 1 and Table 2).

To reveal the bio-function relationship among the proteins, a hierarchical clustering based on Pearson correlation of variances was applied by $\mathrm{R}$ studio. Figure 2 shows the hierarchical clustering of 27 identified proteins, where an increasing red color shows increasing protein expression levels (Figure 2). The most striking area of up-regulation in MPP patients was indicated by a region with a series of protein peaks (red color).

TABLE 2 | Proteins identified following LC-MS/MS of MPP different from healthy and disease control fractions.

\begin{tabular}{|c|c|c|c|c|c|c|c|c|}
\hline Gi number & Protein name & Gene & Uniprot identifier & Mass & pl & Scores & MPP:HC & MPP:IDC \\
\hline gi|50659080 & Alpha-1-antichymotrypsin precursor & SERPINA3 & P01011 & 47.6 & 5.2 & 279.4 & 0.386 & 0.483 \\
\hline gi|7661960 & Kinetochore-associated protein 1 & KNTC1 & P50748 & 250.6 & 5.6 & 127.2 & 0.069 & 0.264 \\
\hline gi|63998985 & Mitogen-activated protein kinase kinase kinase 19 isoform 3 & MAP3К19 & Q56UN5 & 137.5 & 6.7 & 123.6 & 2.174 & 1.961 \\
\hline gi|93204879 & PR domain zinc finger protein 15 isoform 1 & PRDM15 & P57071 & 169.2 & 9.6 & 123.4 & 0.233 & 0.439 \\
\hline gi|31791053 & Zinc finger protein 804B & ZNF804B & A4D1E1 & 152.5 & 9.8 & 116.8 & 0.178 & 0.189 \\
\hline gi|4885583 & rho-associated protein kinase 1 & ROCK1 & Q13464 & 158.1 & 5.6 & 113.3 & 2.941 & 0.431 \\
\hline gi|572882727 & $\begin{array}{l}\text { 1-phosphatidylinositol 4,5-bisphosphate phosphodiesterase } \\
\text { epsilon-1 isoform } 3\end{array}$ & PLCE1 & Q9P212 & 256.9 & 6 & 106.8 & 0.188 & 2.000 \\
\hline gi|6912622 & DNA repair and recombination protein RAD54B isoform 1 & $R A D 54 B$ & Q9Y620 & 102.9 & 9.4 & 98.7 & 0.300 & 8.333 \\
\hline gi|33620769 & E3 ubiquitin-protein ligase RBBP6 isoform 1 & $R B B P 6$ & Q7Z6E9 & 201.4 & 10.2 & 89.3 & 2.174 & 0.529 \\
\hline gi|108773808 & Coiled-coil domain-containing protein 174 & CCDC174 & Q6PII3 & 53.9 & 6 & 84.6 & 0.361 & 0.266 \\
\hline gi|262118216 & Coiled-coil domain-containing protein 88B precursor & CCDC88B & A6NC98 & 164.7 & 4.9 & 83.1 & 2.273 & 0.328 \\
\hline gi|140161498 & $\begin{array}{l}\text { Microtubule-associated tumor suppressor candidate } 2 \\
\text { isoform a }\end{array}$ & MTUS2 & Q5JR59 & 151.1 & 6.3 & 82.3 & 0.503 & 0.526 \\
\hline gi|44921615 & Exocyst complex component 8 & EXOC8 & Q8IYI6 & 81.7 & 5.2 & 78.1 & 2.941 & 0.431 \\
\hline gi|356461016 & Gem-associated protein 5 isoform 2 & GEMIN5 & Q8TEQ6 & 168.4 & 6.2 & 73.6 & 0.439 & 0.526 \\
\hline gi|4502157 & Apolipoprotein C-I precursor & APOC1 & P02654 & 9.3 & 9.3 & 73.5 & 2.083 & 2.273 \\
\hline gi|302129652 & Annexin A6 isoform 2 & ANXA6 & P08133 & 72.4 & 5.3 & 71.3 & 0.154 & 0.103 \\
\hline gi|338753408 & Transcription factor IIIB 90 kDa subunit isoform 5 & $B R F 1$ & Q92994 & 61.8 & 5 & 60.8 & 0.455 & 0.372 \\
\hline gi|32967603 & $\begin{array}{l}\text { Bromodomain adjacent to zinc finger domain protein } 1 \mathrm{~A} \\
\text { isoform a }\end{array}$ & $B A Z 1 A$ & Q9NRL2 & 178.6 & 6.2 & 59.6 & 0.538 & 0.227 \\
\hline gi|321267571 & CASP8 and FADD-like apoptosis regulator isoform 6 & CFLAR & O15519 & 41.3 & 7 & 51.9 & 0.538 & 0.493 \\
\hline gi|83700225 & Potassium-transporting ATPase alpha chain 2 isoform 2 & ATP12A & P54707 & 115.4 & 6.1 & 45.4 & 0.309 & 0.439 \\
\hline gi|390407643 & cAMP-regulated phosphoprotein 21 isoform 4 & ARPP21 & Q9UBL0 & 88.5 & 6.5 & 41.1 & 0.439 & 0.422 \\
\hline gi|284925165 & SUN domain-containing protein 1 isoform c & SUN1 & 094901 & 76.4 & 6.2 & 40.4 & 2.326 & 2.778 \\
\hline gi|4507955 & Transcriptional repressor protein YY1 & $Y Y 1$ & P25490 & 44.7 & 5.8 & 38.1 & 2.128 & 0.124 \\
\hline gi|392050772 & Zinc finger protein 850 isoform 2 & ZNF850 & A0A087X0M6 & 121.8 & 10.1 & 37.5 & 2.041 & 2.128 \\
\hline gi|149588534 & Ataxin-7-like protein 3 isoform b & ATXN7L3 & Q14CW9 & 38.6 & 6.8 & 36.6 & 0.278 & 0.407 \\
\hline gi|222352127 & Protein sidekick-2 precursor & SDK2 & Q58EX2 & 239.2 & 6.6 & 85.3 & 0.483 & 0.546 \\
\hline gi|378925630 & Ubiquitin carboxyl-terminal hydrolase 17-like protein 10 & USP17L10 & C9JJH3 & 59.8 & 9.4 & 50.5 & 0.483 & 0.546 \\
\hline
\end{tabular}




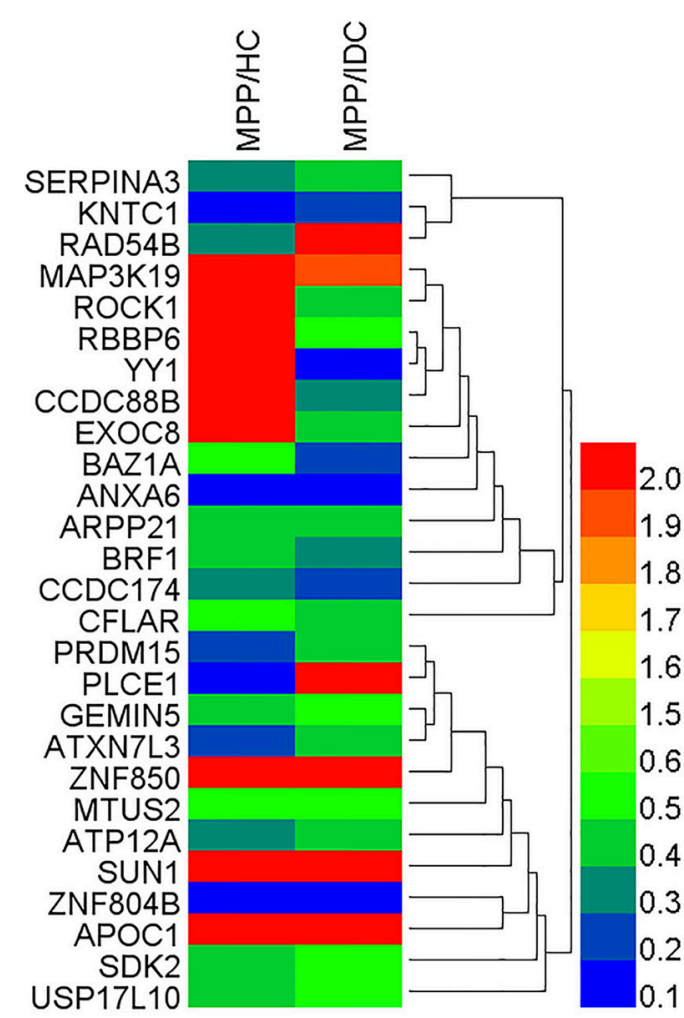

FIGURE 2 | Heat map of the 27 identified proteins. The most striking area of up-regulation in TB patients is seen in the region where a series of protein peaks showed in red.

\section{Bioinformatic Analysis of Differentially Expressed Proteins during MP Infection} GO Analysis

GO annotation was used to analyze the functions of the 27 proteins, which were classified into three categories: CC, MF, and BP. To visualize the annotation of gene sets, WEGO was used to plot the distribution of GO annotations. The differentially identified proteins were subcategorized into 35 main hierarchically structured GO classifications including $9 \mathrm{CC}, 6 \mathrm{MF}$, and $20 \mathrm{BP}$ (Figure 3 and Supplementary Table 4).

The majority of proteins in the CC category had a cellular part and organelle distribution. The 27 proteins in the MF category were mainly related to binding, catalysis, enzyme regulator, molecular transducer, transcription regulator, and transporter. The 27 proteins in the BP category were mainly associated with cellular processes, metabolic processes, cellular component organization, biological regulation, and pigmentation. This analysis indicated that the identified proteins involved in these GO categories might have the most important roles in the MP infection process.

\section{KEGG Enrichment Analysis}

To obtain more information with regards to the markers, a pathway analysis was conducted using KEGG. Using a standard of $P<0.05$ and impact factor threshold $>0$, the pathway analysis results demonstrated that the cAMP signaling pathway $(P=0.026)$ and proteoglycans in cancer $(P=0.028)$ were significantly associated with MPP infection (Figure 4 and Supplementary Table 5).

\section{Identification of Novel Biomarkers for the Differentiate of MPP \\ Validation of Identified Proteins by ELISA}

Based on the bioinformatics analysis, five potential candidate biomarkers (APOC1, SERPINA3, ANXA6, KNTC1, and CFLAR) were selected for verification (Figure 5 and Supplementary Table 6). The selection principles were based on the following standards: (1) high fold change; (2) representative in the 27 proteins; (3) associated with immunity or infectious diseases; and (4) present the same trends when compared with the HC and IDC.

Significant differences in SERPINA3, APOC1, and CFLAR levels were observed among all three groups and their ratios were consistent with the trends of the proteomic results $(P<0.001$, 0.001 , and 0.008, respectively). As shown in Figure 5, the level of APOC1 protein in MPP was markedly increased when compared with that in HC or IDC. In contrast, significantly lower levels of the two proteins (SERPINA3 and CFLAR) were observed in the MPP group compared with that in HC or IDC. Although the levels of ANXA6 and KNTC1 were lower in the MPP group compared with the HC group, there were no differences between the MPP and IDC patients.

\section{Performance of Each Selected Protein to Differentiate of MPP}

The AUC, sensitivity, and specificity of ROC curves calculated at optimal cutoffs for the three different proteins are shown in Table 3. A comparison of MPP patients and HC showed APOC1 had the largest AUC of 0.853 , with $77.6 \%$ sensitivity and $81.1 \%$ specificity. The comparison of MPP and IDC patients showed APOC1 had a relatively high AUC of 0.882 , with $77.6 \%$ sensitivity and $85.3 \%$ specificity. SERPINA3 and CFLAR had AUC-values of 0.739 and 0.524 when MPP patients were compared with $\mathrm{HC}$, respectively, and they had lower AUC-values of 0.643 and 0.647 when compared between MPP and IDC patients, respectively. In addition, we combined the $\mathrm{HC}$ and IDC for further analysis. APOC1, SERPINA3, and CFLAR had AUCvalues of $0.866,0.698$, and 0.578 when MPP was compared with HC plus IDC, respectively. Our results showed that the APOC1 in MPP group presented the best AUC among these three proteins when compare with HC, IDC, or HC combined with IDC.

\section{Sensitivities and Specificities of Combinations of Selected Proteins}

Using the same optimal cutoffs for each protein, we calculated the sensitivities and specificities of combinations of two or three selected proteins to differentiate MPP. As shown in Table 4, although the specificities of combined proteins were generally higher than APOC1, the sensitivities of the combined proteins were lower than this best protein for discriminating MPP patients from HC or IDC patients. Combined with the performance of each selected protein to differentiate of MPP, the results showed that APOC1 alone was the best choice in children. 


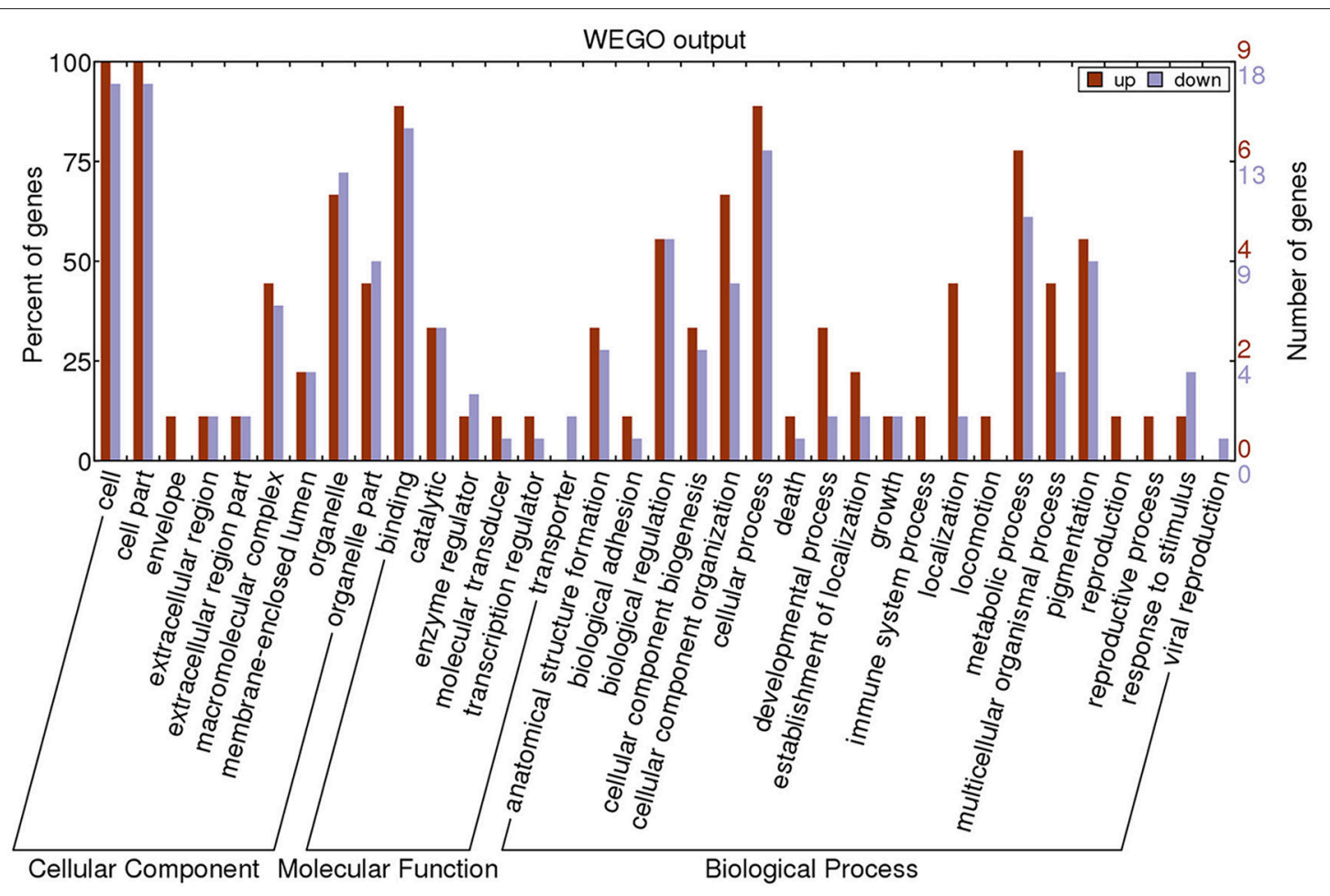

FIGURE 3 | Gene Ontology (GO) classification of differentially expressed proteins by label-free Quantitative Proteomics experiments between MPP and controls. The differentially expressed proteins are grouped into three hierarchically structured GO terms: biological process, cellular component, and molecular function. The $y$-axis indicates the number and percent of proteins in each GO term.

\section{DISCUSSION}

Immune responses, induced by MP infection, have an important effect on pathogenic mechanisms and protein expression. Traditionally, proteins appear in the circulation through many different mechanisms including secretion after subjects are infected by a pathogen, when production is stimulated by antigens, or by direct secretion by MP (Li et al., 2014). Recently, high-throughput technologies have been used to investigate protein expression, and therefore, the accurate detection and quantification of these MP-associated proteins can be used as potential diagnostic markers.

Previous methods used to detect MPs had limitations (Daxboeck et al., 2003; Long et al., 2012; Yuta et al., 2014; Katsushima et al., 2015). In clinical practice, co-infections are common in children with pneumonia. Therefore, in this study, HC and IDC were both used as controls. By using label-free quantitative analysis, we analyzed plasma proteins among MPP, HC, and IDC individuals. This proteomic approach allowed the measurement of changes in protein expression, which might be used to "forecast" disease pathogenesis. As reported here, 282 and 76 proteins were identified in the MPP group compared with the HC and IDC groups, of which 27 were overlapping. In addition, WEGO was used to analysis the function of the 27 different proteins. Here, we observed that a majority of the proteins had a cellular distribution and organelles. They were mainly related to binding, catalysis, enzyme regulator, molecular transducer, transcription regulator, and transporter. They mostly participated in cellular processes, metabolic processes, cellular component organization, biological regulation, and pigmentation, all of which are thought to be essential in the pathogenesis of MP.

Among the 27 proteins identified, five potential candidate biomarkers (SERPINA3, KNTC1, APOC1, ANXA6, and CFLAR) were selected for verification because they correlated with immune responses in other infectious diseases. However, ELISA showed that only the levels of SERPINA3, APOC1, and CFLAR were significantly different among all three groups and their ratios were consistent with the trends of the proteomics analyses. Using the same optimal cutoffs for each protein, we analyzed the sensitivities and specificities of different combinations of two or three of the different proteins for the diagnosis of MPP. The specificities of combined proteins were generally higher than APOC1, while the sensitivities of combined proteins were generally lower than the best protein for differentiating MPP patients and $\mathrm{HC}$ or IDC patients. In summary, our results showed that APOC1 alone was the best choice for differentiate of MPP in children. APOC1 had the best sensitivity and specificity. To the best of our knowledge, the sensitivity and specificity of the MP-specific antigens currently used are poor when used as a diagnostic test based on ineffective antibody detection (Zhang et al., 2014). Li et al. (2014) used label-free quantitative proteomics to demonstrate that IL-33 production in A549 cells was increased when they were infected with MP. They also found 


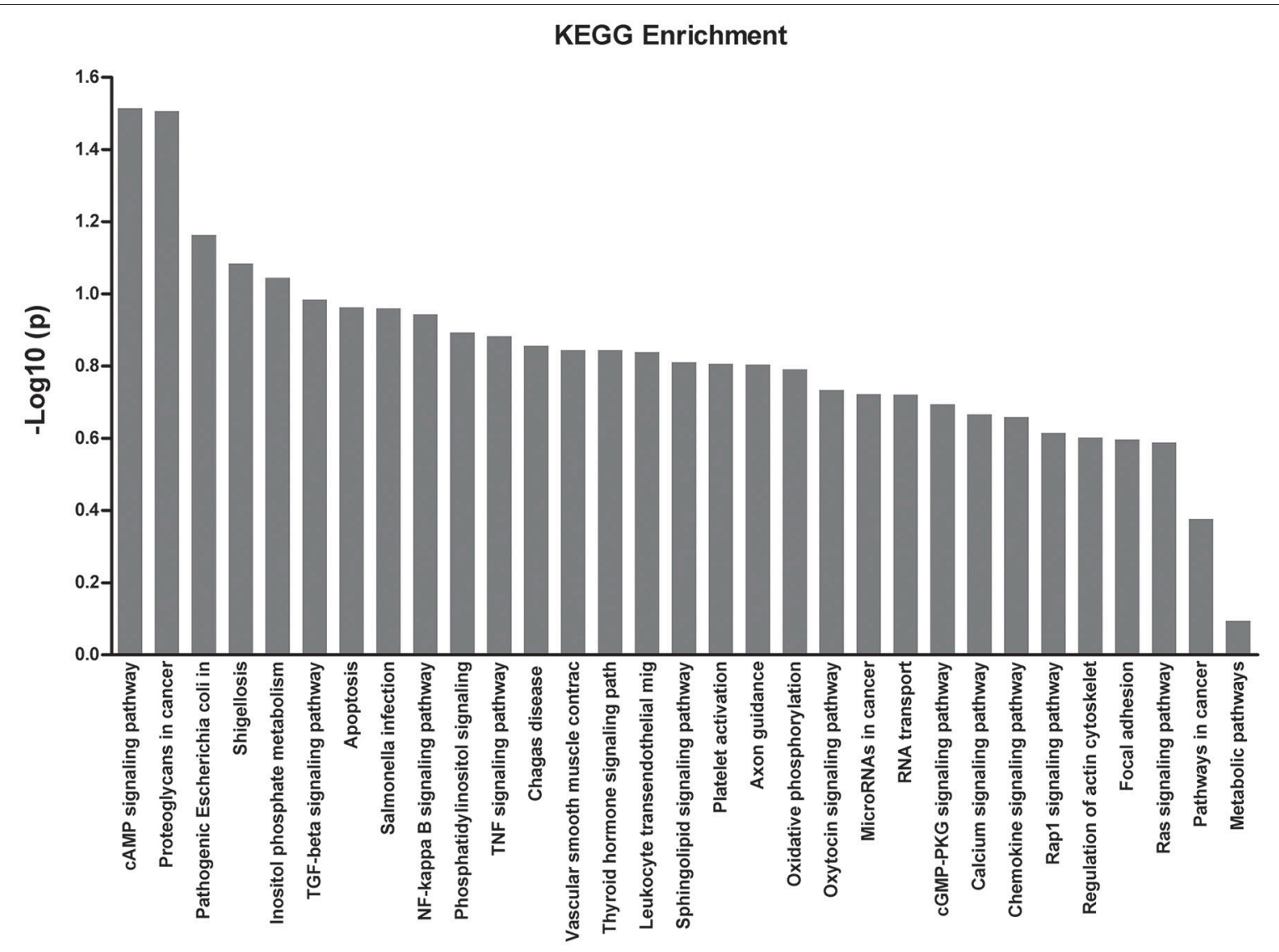

FIGURE 4 | KEGG enrichment analysis of the differential expressed proteins.

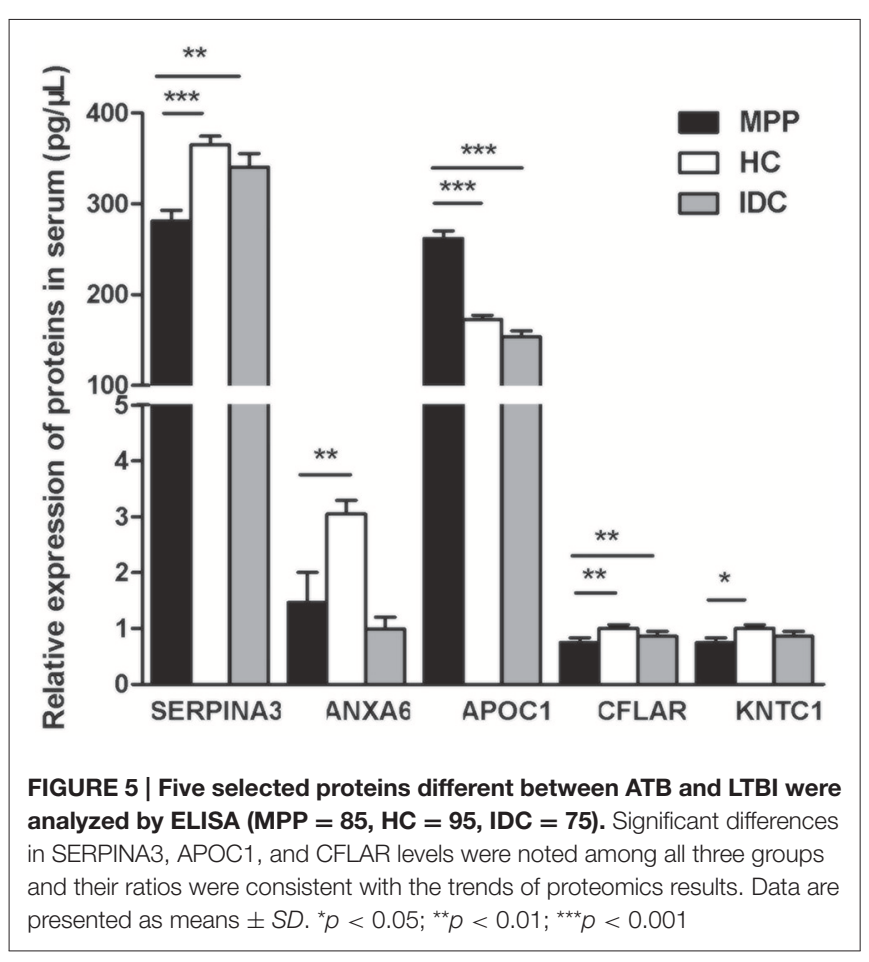

that IL-33 levels were higher in MPP patients with sensitivity and specificity accuracy of 70.0 and $73.3 \%$, respectively (Li et al., 2014). However, larger sample sizes are needed for further identification and quantification.

MP is a unique cell wall deficient and cholesterol requiring bacteria (Berbée et al., 2005; Ko et al., 2014). Cholesterol metabolism is essential for MP growth and infection. APOC1 is a $6.6-\mathrm{kDa}$ plasma protein that inhibits receptor-mediated lipoprotein clearance (Shulman et al., 1975). It is secreted by many organs, including the liver, spleen, and lung, and is secreted into the blood (Lauer et al., 1988). Therefore, the plasma level of APOC1 is associated with cholesterol metabolism and MP infection status in humans. Lipid metabolism is one of the essential changes in many infectious diseases, including MPP (Katsel et al., 2007; Xiang et al., 2007). Furthermore, many cholesterol metabolites have been analyzed by studies with the aim of screening and identifying early biomarkers for infectious disease (Leoni et al., 2006; Bach et al., 2009; Gilch et al., 2009). Several genes associated with cholesterol metabolism and lipid biosynthesis were reported to be up-regulated in serum or plasma (Brown et al., 2005). Because APOC1 activates cholesterol metabolism (Barbisin et al., 2014), its up-regulation leads to an increase in cholesterol biosynthesis, consistent with the concomitant presence of MP infectious disease. 
TABLE 3 | AUC, sensitivity, and specificity for ROC curves calculated at optimal cutoff for SERPINA3, APOC1, and CFLAR.

\begin{tabular}{|c|c|c|c|c|c|c|}
\hline Significant proteins by comparison groups & AUC & $95 \% \mathrm{Cl}$ & Sensitivity (\%) & Specificity (\%) & $P$-value & $\log _{2} F C$ \\
\hline \multicolumn{7}{|l|}{ MPP vs. HC } \\
\hline APOC1 & 0.853 & $0.796-0.907$ & 77.6 & 81.1 & 8.81E-18 & 0.56 \\
\hline SERPINA3 & 0.739 & $0.662-0.818$ & 77.6 & 68.4 & $1.38 \mathrm{E}-7$ & -0.34 \\
\hline CFLAR & 0.524 & $0.436-0.61$ & 77.6 & 42.1 & $7.56 \mathrm{E}-3$ & 0.12 \\
\hline \multicolumn{7}{|l|}{ MPP vs. IDC } \\
\hline APOC1 & 0.882 & $0.834-0.93$ & 77.6 & 85.3 & $1.37 \mathrm{E}-18$ & 0.78 \\
\hline SERPINA3 & 0.643 & $0.558-0.735$ & 72.9 & 65.3 & $2.15 \mathrm{E}-3$ & -0.33 \\
\hline CFLAR & 0.647 & $0.55-0.733$ & 81.2 & 50.7 & $1.54 \mathrm{E}-3$ & -0.66 \\
\hline \multicolumn{7}{|l|}{ MPP vs. (HC + IDC) } \\
\hline APOC1 & 0.866 & $0.816-0.915$ & 77.6 & 82.9 & 1.34E-26 & 0.55 \\
\hline SERPINA3 & 0.698 & $0.624-0.763$ & 77.6 & 64.7 & $1.42 \mathrm{E}-6$ & -0.05 \\
\hline CFLAR & 0.578 & $0.514-0.648$ & 71.8 & 54.1 & $3.74 \mathrm{E}-3$ & -0.64 \\
\hline
\end{tabular}

TABLE 4 | Sensitivities and specificities of combinations of two or three proteins for differentiation between MPP and HC, IDC, or HC + IDC.

\begin{tabular}{|c|c|c|c|c|c|c|}
\hline \multirow[t]{2}{*}{ Proteins } & \multicolumn{2}{|c|}{ MPP vs. HC } & \multicolumn{2}{|c|}{ MPP vs. IDC } & \multicolumn{2}{|c|}{ MPP vs. HC + IDC } \\
\hline & Sensitivity (\%) & Specificity (\%) & Sensitivity (\%) & Specificity (\%) & Sensitivity (\%) & Specificity (\%) \\
\hline APOC1 + SERPINA3 & 76.5 & 87.4 & 76.5 & 85.3 & 77.7 & 82.9 \\
\hline APOC1 + CFLAR & 75.3 & 82.1 & 76.5 & 81.3 & 75.3 & 81.8 \\
\hline SERPINA3 + CFLAR & 75.3 & 68.4 & 74.1 & 58.7 & 75.3 & 67.1 \\
\hline SERPINA3 + APOC1 + CFLAR & 74.1 & 84.2 & 78.8 & 81.3 & 77.7 & 83.5 \\
\hline
\end{tabular}

Our study demonstrated that APOC1 alone is a potential biomarker for differentiate of MPP in children. This was an exploratory study to detect novel plasma proteomics biomarkers and further studies are required. MPP causes up to $40 \%$ of CAP in children and biomarker selection requires a large sample verification. Clearly, this should be the focus of further investigations.

In conclusion, 27 potential MPP plasma biomarkers were identified using LC-MS/MS. After ELISA verification, APOC1 was identified with satisfactory sensitivity and specificity to discriminate MPP from HC or IDC, and represents a viable approach for the differentiate of MPP.

\section{AUTHOR CONTRIBUTIONS}

JL and AS designed the experiments. JL and LS performed the experiments and analyzed the data. FX, WJ, and HQ provided technical support for the experiments. AS, JX, and CS provided comments and technical advice. JL, LS, and AS wrote the manuscript. JL, BX, and QL revised the manuscript. All authors discussed the results, commented on the manuscript, and agreed to be accountable for all aspects of the work in

\section{REFERENCES}

Agranoff, D., Fernandez-Reyes, D., Papadopoulos, M. C., Rojas, S. A., Herbster, M., Loosemore, A., et al. (2006). Identification of diagnostic markers for tuberculosis by proteomic fingerprinting ensuring that questions related to the accuracy or integrity of any part of the work are appropriately investigated and resolved.

\section{FUNDING}

This work was supported by grants from Beijing Natural Science Foundation (No. 7164257), Capital Health Research and Development of Special Grant (No. 2016-1-2092), the BeiJing Talents Fund (No. 2014000021469G244), and collaborative Innovation Center of Infectious Diseases (No. PXM2014_014226_000011).

\section{ACKNOWLEDGMENTS}

We thank all the authors in this manuscript.

\section{SUPPLEMENTARY MATERIAL}

The Supplementary Material for this article can be found online at: http://journal.frontiersin.org/article/10.3389/fmicb. 2016.01961/full\#supplementary-material

of serum. Lancet 368, 1012-1021. doi: 10.1016/S0140-6736(06) 69342-2

Bach, C., Gilch, S., Rost, R., Greenwood, A. D., Horsch, M., Hajj, G. N., et al. (2009). Prion-induced activation of cholesterogenic gene expression by Srebp2 in neuronal cells. J. Biol. Chem. 284, 31260-31269. doi: 10.1074/jbc.M109.004382 
Barbisin, M., Vanni, S., Schmädicke, A. C., Montag, J., Motzkus, D., Opitz, L., et al. (2014). Gene expression profiling of brains from bovine spongiform encephalopathy (BSE)-infected cynomolgus macaques. BMC Genomics 15:434. doi: 10.1186/1471-2164-15-434

Berbée, J. F., Havekes, L. M., and Rensen, P. C. (2005). Apolipoproteins modulate the inflammatory response to lipopolysaccharide. J. Endotoxin Res. 11, 97-103. doi: 10.1179/096805105X35215

Brown, A. R., Rebus, S., McKimmie, C. S., Robertson, K., Williams, A., and Fazakerley, J. K. (2005). Gene expression profiling of the preclinical scrapie-infected hippocampus. Biochem. Biophys. Res. Commun. 334, 86-95. doi: 10.1016/j.bbrc.2005.06.060

Chen, Z. R., Zhang, G. B., Wang, Y. Q., Yan, Y. D., Zhou, W. F., Zhu, C. H., et al. (2013). Soluble B7-H3 elevations in hospitalized children with Mycoplasma pneumoniae pneumonia. Diagn. Microbiol. Infect. Dis. 77, 362-366. doi: 10.1016/j.diagmicrobio.2013.09.006

Covani, U., Marconcini, S., Giacomelli, L., Sivozhelevov, V., Barone, A., and Nicolini, C. (2008). Bioinformatic prediction of leader genes in human periodontitis. J. Periodontol. 79, 1974-1983. doi: 10.1902/jop.2008.080062

Daxboeck, F., Krause, R., and Wenisch, C. (2003). Laboratory diagnosis of Mycoplasma pneumoniae infection. Clin. Microbiol. Infect. 9, 263-273. doi: 10.1046/j.1469-0691.2003.00590.x

Engwegen, J. Y., Gast, M. C., Schellens, J. H., and Beijnen, J. H. (2006). Clinical proteomics: searching for better tumor markers with SELDI-TOF mass spectrometry. Trends Pharmacol. Sci. 27, 251-259. doi: 10.1016/j.tips.2006.03.003

Gilch, S., Bach, C., Lutzny, G., Vorberg, I., and Schatzl, H. M. (2009). Inhibition of cholesterol recycling impairs cellular $\mathrm{PrP}^{\mathrm{Sc}}$ propagation. Cell. Mol. Life Sci. 66, 3979-3991. doi: 10.1007/s00018-009-0158-4

Hodgetts, A., Levin, M., Kroll, J. S., and Langford, P. R. (2007). Biomarker discovery in infectious diseases using SELDI. Future Microbiol. 2, 35-49. doi: $10.2217 / 17460913.2 .1 .35$

Hong, M., Zhang, X., Hu, Y., Wang, H., He, W., Mei, H., et al. (2009). The potential biomarkers for thromboembolism detected by SELDI-TOF-MS. Thromb. Res. 123, 556-564. doi: 10.1016/j.thromres.2008.05.019

Kanehisa, M., Goto, S., Kawashima, S., Okuno, Y., and Hattori, M. (2004). The KEGG resourcefor deciphering the genome. Nucleic Acids Res. 32, 277-280. doi: 10.1093/nar/gkh063

Katsel, P., Li, C., and Haroutunian, V. (2007). Gene expression alterations in the sphingolipid metabolism pathways during progression of dementia and Alzheimer's disease: a shift toward ceramide accumulation at the earliest recognizable stages of Alzheimer's disease? Neurochem. Res. 32, 845-856. doi: 10.1007/s11064-007-9297-x

Katsushima, Y., Katsushima, F., Suzuki, Y., Seto, J., Mizuta, K., Nishimura, H., et al. (2015). Characteristics of Mycoplasma pneumoniae infection identified on culture in a pediatric clinic. Pediatr. Int. 57, 247-252. doi: 10.1111/ped.12513

Ko, H. L., Wang, Y. S., Fong, W. L., Chi, M. S., Chi, K. H., and Kao, S. J. (2014). Apolipoprotein C1 (APOC1) as a novel diagnostic and prognostic biomarker for lung cancer: a marker phase I trial. Thorac. Cancer 5, 500-508. doi: $10.1111 / 1759-7714.12117$

Lauer, S. J., Walker, D., Elshourbagy, N. A., Reardon, C. A., Levy-Wilson, B., and Taylor, J. M. (1988). Two copies of the human apolipoprotein C-I gene are linked closely to the apolipoprotein E gene. J. Biol. Chem. 263, 7277-7286.

Lee, J., Kim, S. H., Choi, D. S., Lee, J. S., Kim, D. K., Go, G., et al. (2015). Proteomic analysis of extracellular vesicles derived from Mycobacterium tuberculosis. Proteomics 15, 3331-3337. doi: 10.1002/pmic.201500037

Leoni, V., Shafaati, M., Salomon, A., Kivipelto, M., Bjorkhem, I., and Wahlund, L. O. (2006). Are the CSF levels of 24S-hydroxycholesterol a sensitive biomarker for mild cognitive impairment? Neurosci. Lett. 397, 83-87. doi: 10.1016/j.neulet.2005.11.046

Li, S., Li, X., Wang, Y., Yang, J., Chen, Z., and Shan, S. (2014). Global secretome characterization of A549 human alveolar epithelial carcinoma cells during Mycoplasma pneumoniae infection. BMC Microbiol. 7:27. doi: 10.1186/1471-2180-14-27

Long, S. S., Pickering, L. K., and Prober, C. G. (2012). Principles and Practice of Pediatric Infectious Diseases, 4th Edn. London: Elsevier Churchill Livingstone.

Meyer Sauteur, P. M., Jacobs, B. C., Spuesens, E. B., Jacobs, E., Nadal, D., Vink, C., et al. (2014). Antibody responses to Mycoplasma pneumoniae: role in pathogenesis and diagnosis of encephalitis? PLoS Pathog. 10:e1003983. doi: 10.1371/journal.ppat.1003983

Papadopoulos, M. C., Abel, P. M., Agranoff, D., Stich, A., Tarelli, E., Bell, B. A., et al. (2004). A novel and accurate test for Human African Trypanosomiasis. Lancet 363, 1358-1363. doi: 10.1016/S0140-6736(04)16046-7

Pinet, F., Beseme, O., Cieniewski-Bernard, C., Drobecq, H., Jourdain, S., Lamblin, N., et al. (2008). Predicting left ventricular remodeling after a first myocardial infarction by plasma proteome analysis. Proteomics $8,1798-1808$. doi: 10.1002/pmic.200700781

Ren, Y., He, Q. Y., Fan, J., Jones, B., Zhou, Y., Xie, Y., et al. (2004). The use of proteomics in the discovery of serum biomarkers from patients with severe acute respiratory syndrome. Proteomics 4, 3477-3484. doi: $10.1002 /$ pmic. 200400897

Sanchez-Vargas, F. M., and Gomez-Duarte, O. G. (2008). Mycoplasma pneumoniae-an emerging extra-pulmonary pathogen. Clin. Microbiol. Infect. 14, 105-117. doi: 10.1111/j.1469-0691.2007.01834.x

Shu, L. H., Lu, Q., Han, L. Y., and Dong, G. H. (2015). SP-D, KL-6, and HTI-56 levels in children with Mycoplasma pneumoniae pneumonia. Int. J. Clin. Exp. Pathol. 8, 11185-11191.

Shulman, R. S., Herbert, P. N., Wehrly, K., and Fredrickson, D. S. (1975). Thf complete amino acid sequence of C-I (apoLp-Ser), an apolipoprotein from human very low density lipoproteins. J. Biol. Chem. 250, 182-190.

Sun, G., Xu, X., Wang, Y., Shen, X., Chen, Z., and Yang, J. (2008). Mycoplasma pneumonia infection induces reactive oxygen species and DNA damage in A549 human lung carcinoma cells. Infect. Immun. 76, 4405-4413. doi: 10.1128/IAI.00575-08

Tamura, A., Matsubara, K., Tanaka, T., Nigami, H., Yura, K., and Fukaya, T. (2008). Methylprednis-olone pulse therapy for refractory Mycoplasma pneumoniae pneumonia in children. J. Infect. 57, 223-228. doi: 10.1016/j.jinf.2008.06.012

Waites, K., and Talkington, D. (2004). Mycoplasma pneumoniae and its role as a human pathogen. Clin. Microbiol. Rev. 17, 697-728. doi: 10.1128/CMR.17.4.697-728.2004

Wang, M., Wang, Y., Yan, Y., Zhu, C., Huang, L., Shao, X., et al. (2014). Clinical and laboratory profiles of refratory Mycoplasma penumoniae pneumonia in children. Int. J. Infec. Dis. 29, 18-23. doi: 10.1016/j.ijid.2014.07.020

Wu, L., Tian, L., Wang, S., Zhang, J., Liu, P., Tian, Z., et al. (2016). Comparative proteomic analysis of the response of maize (Zea mays L.) leaves to long photoperiod condition. Front. Plant. Sci. 7:752. doi: 10.3389/fpls.2016. 00752

Xiang, W., Hummel, M., Mitteregger, G., Pace, C., Windl, O., Mansmann, U., et al. (2007). Transcriptome analysis reveals altered cholesterol metabolism during the neurodegeneration in mouse scrapie model. J. Neurochem. 102, 834-847. doi: 10.1111/j.1471-4159.2007.04566.x

Yuta, A., Tomohiro, O., Shinya, T., Tetsuo, T., and Akihiko, S. (2014). Clinical utility of loop-mediated isothermal amplification for rapid diagnosis of Mycoplasma pneumoniae in children. J. Med. Microbiol. 63, 248-251. doi: 10.1099/jmm.0.068288-0

Zhang, X., Guo, T., Wang, H., He, W., Mei, H., Hong, M., et al. (2008). Potential biomarkers of acute cerebral infarction detected by SELDITOF-MS. Am. J. Clin. Pathol. 130, 299-304. doi: 10.1309/CA242R5VY1 4HUGE8

Zhang, X., Li, W., Hou, Y., Niu, Z., Zhong, Y., Zhang, Y., et al. (2014). Comparative membrane proteomic analysis between lung adenocarcinoma and normal by iTRAQ labeling mass spectrometry. Am. J. Transl. Res. 6, 267-280.

Conflict of Interest Statement: The authors declare that the research was conducted in the absence of any commercial or financial relationships that could be construed as a potential conflict of interest.

Copyright (C) $2016 \mathrm{Li}$, Sun, Xu, Qi, Shen, Jiao, Xiao, Li, Xu and Shen. This is an open-access article distributed under the terms of the Creative Commons Attribution License (CC BY). The use, distribution or reproduction in other forums is permitted, provided the original author(s) or licensor are credited and that the original publication in this journal is cited, in accordance with accepted academic practice. No use, distribution or reproduction is permitted which does not comply with these terms. 